\section{Evaluación de síntomas no motores y descontrol de impulsos en usuarios con enfermedad de Parkinson por el médico en atención primaria}

\author{
PHILIPPE SALLES GÁNDARA ${ }^{1,2}$, PEDRO CHANÁ-CUEVAS ${ }^{2,3}$

\section{Nonmotor symptoms in 32 patients with Parkinson disease}

Background: Nonmotor symptoms of Parkinson disease significantly hamper the quality of life of patients and have prognostic significance. Aim: To evaluate the presence of nonmotor symptoms in patients with Parkinson disease. Material and Methods: A structured interview was carried out in 32 patients aged $74 \pm 9$ years (53\% men) with Parkinson disease asking specifically for impulse control disorders and dopaminergic dysregulation. The following scales were also applied: Hoehn \& Yahr scale, Montreal Cognitive Assessment, Geriatric depression scale, Nonmotor symptom scale and REM sleep scale. Results: A high frequency of nonmotor symptoms was recorded, specially mood, sleep, urinary and gastrointestinal problems and impulse control disorders. Conclusions: Nonmotor symptoms must be actively sought and managed in patients with Parkinson disease.

(Rev Med Chile 2020; 148: 1075-1082)

Key words: Depression; Disruptive, Impulse Control, and Conduct Disorders; Parkinson Disease; Physicians, Primary Care; Primary Health Care.

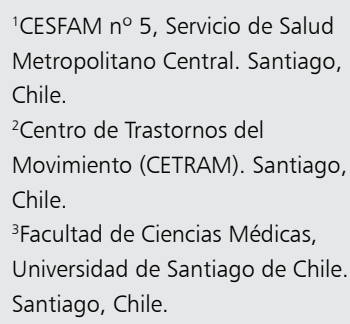

Trabajo no recibió financiamiento Los autores declaran no tener conflictos de interés.

Correspondencia a: Philippe Salles Gándara Belisario Prats 1597-B, Independencia. Santiago, Chile. philippe.salles.g@gmail.com

\section{L} a Enfermedad de Parkinson (EP) es la segunda patología neurodegenerativa más frecuente después de la enfermedad de Alzheimer. Su prevalencia se estima en 1 a 2 por 1.000 habitantes, afectando $1 \%$ de la población sobre 60 años $^{1}$. En Chile de acuerdo a cifras internacionales el año 2013 se estimaba cerca de 40.000 afectados $^{2}$. La EP probable se diagnostica por criterios clínicos que consideran la presencia de síntomas motores de parkinsonismo ${ }^{3}$. En las últimas décadas ha ganado atención la relevancia y frecuencia de síntomas no motores $(\mathrm{SNM})^{4}$; Estas manifestaciones son frecuentes, desde estadíos prodrómicos, en todas las etapas de la enfermedad $^{5}$. Su prevalencia y severidad se incrementa con la progresión, así como también cambian los dominios afectados ${ }^{6}$. Los pacientes reportan generalmente compromiso de varios dominios de SNM, siendo particularmente prevalentes los síntomas genitourinarios, cognitivos, autonómicos, sexuales y problemas de sueño ${ }^{7}$. Los SNM determinan un impacto incluso mayor que los síntomas motores sobre la calidad de vida en pacientes con $\mathrm{EP}^{8}$. Así como existen subtipos motores de la EP (predominio temblor, inestabilidad postural/alteración de la marcha, o mixtos) $)^{9}$, algunos autores han propuesto relacionar los grupos de dominios de SNM afectados con probables biomarcadores, estableciendo los subtipos de "tronco, límbico y cortical" 10 . Se ha establecido que la presencia de síntomas cognitivos y genitourinarios en etapas tempranas pueden ser consideradas marcadores de progresión más rápida de la enfermedad estableciendo un peor pronóstico ${ }^{11}$. 
Los trastornos del descontrol de impulsos son definidos como conductas repetitivas, excesivas y compulsivas, en un nivel que interfiere con el normal funcionamiento de una persona. Existe creciente evidencia y preocupación por el riesgo de pacientes con EP de desarrollar este tipo de conductas. Entre las más reconocidas se encuentran 1) Juego patológico; 2) Compras compulsivas; 3) Conductas sexuales; 4) Apetito urgente o craving; 5) Punding, conductas repetitivas ritualísticas sin propósito con intensa preocupación por actividades específicas (coleccionismo, orden, reposicionar objetos); 6) Hobismo, con conductas de nivel más complejo (ej. Ejercicio excesivo, uso de internet, lectura, trabajo artístico, trabajo en proyectos); 7) Coleccionismo; 8) Caminatas extensas sin rumbo. También se considera dentro de este grupo de SNM de espectro conductual el uso excesivo de fármacos anti parkinsonianos o síndrome de disregulación dopaminérigica. En algunos casos consecuentemente generan serios problemas financieros, legales y psicosociales, con gran impacto en la calidad de vida ${ }^{12}$. Los trastornos del control de impulso han sido reportados en $20 \%$ de pacientes con EP sin tratamiento, sin embargo está fuertemente asociado con el uso de agonistas dopaminérgicos, de manera dosis dependiente, llegando a una prevalencia de más de $50 \%$ en algunos trabajos ${ }^{13}$. A pesar de ser bien reconocidos en la literatura, en la práctica clínica son sub-diagnosticados $y$, por lo tanto, inadecuadamente manejados, esto se explica en parte por la pobre conciencia y reporte del fenómeno por parte de los pacientes, surgiéndose aplicar entrevistas estructuradas para mejorar su pesquisa al paciente y familiar o cuidador cuando corresponde ${ }^{13}$.

La guía clínica MINSAL del año 2010 sugiere que el médico en atención primaria de salud (APS) debe sospechar la EP y derivar oportunamente al nivel secundario para su confirmación. Luego a nivel primario el médico cumple la función de monitorizar síntomas atípicos y efectos adversos a fármacos, además del estado de salud general del enfermo (Guía Clínica Enfermedad de Parkinson, MINSAL 2010).

Con el fin de identificar las principales manifestaciones no motoras de los pacientes con EP del CESFAM no 5 e intentar establecer estrategias efectivas y complementarias en la evaluación y monitorización de estos síntomas, se llevo a cabo el presente trabajo.

\section{Materiales y Métodos}

Para describir la sintomatología no motora y entender las necesidades de cuidado de los pacientes con EP del CESFAM número 5, del Servicio de Salud Metropolitano Central, se realizó un estudio descriptivo de diseño transversal. Se evaluaron 32 de un total de 40 pacientes inscritos para el retiro de fármacos anti parkinsonianos al año 2012 en la farmacia de este centro, quienes fueron invitados a participar voluntariamente mediante citación telefónica a una evaluación médica estandarizada por un médico general del centro de salud (autor de este trabajo), quien contó con la asesoría del centro de trastornos del movimiento (CETRAM), y la aprobación del comité de ética de CETRAM. Todos los pacientes fueron diagnosticados previamente por un neurólogo en el Hospital San Borja Arriarán, centro en que mantenían controles regulares. Cuatro de los 40 inscritos, pertenecían al programa de atención domiciliaria por dependencia severa, motivo por el que no fueron incorporados al estudio, otros 4 pacientes no pudieron ser contactados.

Para determinar la representatividad de nuestra muestra calculamos un tamaño muestral de 31 pacientes, tomando como población conocida a 36 pacientes (habiendo excluido a aquellos pertenecientes al programa de dependencia severa), considerando un nivel de confianza de $95 \%$ y margen de error de $7 \%$.

Para el registro de la información se diseñó una entrevista estandarizada incorporando variables demográficas y las siguientes herramientas de evaluación:

1) Escala Hoehn \& Yahr ${ }^{16,17}$.

2) Test de MoCA (Montreal Cognitive Assessment) en su versión en español, cuya instrucción de uso se encuentra en internet, y ha demostrado utilidad como instrumento de screening para detección de deterioro cognitivo leve o demencia en EP, validada en población chilena con un punto de corte $<21$ para deterioro cognitivo leve $\mathrm{y}<20$ para demencia leve ${ }^{18-21}$.

3) Escala de Depresión Geriátrica en su versión abreviada de 15 preguntas GDS-15, validada al español para su uso en APS, y como herramienta de screening de depresión en pacientes con $\mathrm{EP}^{22-24}$.

4) Escala de síntomas no motores desarrollada $y$ validada por el grupo de estudio de SNM, dis- 
ponible en español (enlace: http://91.121.70.71/ docu/Escala_de_evaluacion_de_sintomas_no_ motores_E_Parkinson.pdf). Esta escala de 30 preguntas aborda 9 diferentes dominios: Sistema cardiovascular/caídas, sueño/fatiga, estado de ánimo/apatía, problemas perceptivos/alucinaciones, atención/memoria, tracto gastrointestinal, función urinaria, función sexual y misceláneo. Cada ítem se puntúa multiplicando el puntaje determinado para la gravedad del síntoma $(0=$ Ninguna; $1=$ Leve; $2=$ Moderada; $3=$ Grave) y su frecuencia de presentación ( $1=$ Raramente; 2 = Ocasional; $3=$ Frecuente; 4 = Muy Frecuente). La puntuación de cada dominio se obtiene sumando el resultado de [gravedad] x [frecuencia] de los ítems que lo componen. El rango teórico del puntaje total es 0-360 puntos. Para esta escala se han propuesto 5 niveles de carga de SNM de acuerdo al puntaje total: Nivel $0(\mathrm{No})=0$; Nivel $1($ Leve $)=1-20 ;$ Nivel $2($ Moderado $)=21-40$; Nivel 3 (Severo) $=41$ - 70; Nivel 4 (Muy severo) $=\geq 71^{25-28}$.

5) Trastorno conductual del sueño REM: Se evaluó interrogando al paciente y/o acompañante sobre la presencia en el mes previo de pesadillas o sueños de contenido violento y conductas motoras violentas "como golpes" durante el sueño, con o sin interrupción de este $e^{29-30}$.

6) Síntomas de descontrol de impulsos y síndrome de desregulación dopaminérgica: Se interrogó dirigidamente sobre la presencia de hipersexualidad, ludopatía, compulsión por comer o craving, compras compulsivas, obsesión por el orden, hobismo, punding y disregulación dopaminérigica. Se incorporó también la presencia de caídas en el último año.

Los resultados se expresaron en números absolutos, porcentajes o medias \pm desviaciones estándar según correspondía.

\section{Resultados}

Los 32 pacientes, 17 de sexo masculino y 15 femenino, completaron todas las evaluaciones establecidas por protocolo. En la Tabla 1 se resumen las características demográficas y comorbilidades de los pacientes evaluados. Veintisiete (84\%) de los pacientes presentaban algún tipo de comorbilidad.
Tabla 1. Variables demográficas y comorbilidades

\begin{tabular}{|c|c|}
\hline Género (hombres) & $17(53,12 \%)$ \\
\hline Edad (años) & $\begin{array}{l}73,75 \pm 8,54 \\
(54-90)\end{array}$ \\
\hline Años de estudio & $9 \pm 4,26(0-16)$ \\
\hline Trabajo remunerado & $10(31,25 \%)$ \\
\hline $\begin{array}{l}\text { Estado civil } \\
\text { - Casado(a) } \\
\text { - Viudo(a) } \\
\text { - Separado(a) } \\
\text { - Soltero(a) }\end{array}$ & $\begin{aligned} 18 & (56,25 \%) \\
9 & (28,12 \%) \\
3 & (9,375 \%) \\
2 & (6,25 \%)\end{aligned}$ \\
\hline $\begin{array}{l}\text { Número de personas en el hogar } \\
\text { (cuidadores) } \\
-\quad \geq 2 \\
-1 \\
-0\end{array}$ & $\begin{array}{r}11 \\
15 \\
7\end{array}$ \\
\hline Antecedente familiar de EP & $6(18,75 \%)$ \\
\hline Edad al diagnóstico de EP (años) & $68,72 \pm 9(51-85)$ \\
\hline $\begin{array}{l}\text { Tiempo de evolución desde } \\
\text { el diagnóstico (años) }\end{array}$ & $5 \pm 3,73(0-16)$ \\
\hline $\begin{array}{l}\text { Sintomatología predominante al } \\
\text { inicio de la enfermedad } \\
\text { - Temblor } \\
\text { - Sd. Akineto-rígido }\end{array}$ & $\begin{array}{l}22(68,75 \%) \\
10(31,25 \%)\end{array}$ \\
\hline $\begin{array}{l}\text { Tratamiento } \\
\text { - Levodopa }(n) \\
\text { o Dosis }\end{array}$ & $\begin{array}{l}31 \\
575 \text { mg/día } \pm 215 \\
67 \text { (300-1.000) }\end{array}$ \\
\hline $\begin{array}{c}\text { - Pramipexol (n) } \\
\text { o Dosis }\end{array}$ & $\begin{array}{l}13 \\
1,38 \mathrm{mg} / \mathrm{día} \pm \\
0,43(0,75-2)\end{array}$ \\
\hline $\begin{array}{c}\text { - Amantadina (n) } \\
\text { o Dosis }\end{array}$ & $\begin{array}{c}1 \\
200 \text { mg/día }\end{array}$ \\
\hline $\begin{array}{c}\text { - Quetiapina (n) } \\
\text { o Dosis }\end{array}$ & $\begin{array}{l}3 \\
25 \mathrm{mg} / \mathrm{dí} a \pm 0\end{array}$ \\
\hline $\begin{array}{l}\text { Participación en rehabilitación } \\
\text { kinésica en APS }\end{array}$ & $6(18,75 \%)$ \\
\hline Pacientes con $\geq 1$ caída último año & $18(56,25 \%)$ \\
\hline $\begin{array}{l}\text { Uso de ayudas técnicas } \\
\text { - Bastón } \\
\text { - Andador } \\
\text { - Silla de ruedas }\end{array}$ & $\begin{array}{rr}13 & (40,62 \%) \\
9 & (28,12 \%) \\
1 & (3,12 \%) \\
3 & (9,37 \%)\end{array}$ \\
\hline $\begin{array}{l}\text { Sin control por especialista }>12 \\
\text { meses }\end{array}$ & $5(15,62 \%)$ \\
\hline $\begin{array}{l}\text { Comorbilidades } \\
\text { - HTA } \\
\text { - DM2 } \\
\text { - Dislipidemia } \\
\text { - Hipotiroidismo } \\
\text { - Artrosis } \\
\text { - Sd. hombro doloroso } \\
\text { - Hipoacusia } \\
\text { - Patología prostática* } \\
\text { - Patología cardiaca** } \\
\text { - Depresión*** } \\
\text { - Tabaquismo detenido/activo }\end{array}$ & $\begin{array}{c}17(53,12 \%) \\
7(21,87 \%) \\
7(21,87 \%) \\
4(12,5 \%) \\
5(15,62 \%) \\
5(15,62 \%) \\
3(9,37 \%) \\
5(15,62 \%) \\
7(21,87 \%) \\
3 \quad(9,37 \%) \\
10 / 1\end{array}$ \\
\hline
\end{tabular}


Tabla 2. Evaluación del compromiso motor de acuerdo a la escala de Hoehn \& Yahr

\begin{tabular}{|c|c|c|}
\hline 1 & Compromiso unilateral, discapacidad ausente o mínima & $3(9,37 \%)$ \\
\hline 2 & Compromiso bilateral o axial, sin alteración del equilibrio & $12(37,5 \%)$ \\
\hline 3 & $\begin{array}{l}\text { Compromiso bilateral, discapacidad leve a moderada. Con alteración de reflejos posturales, indepen- } \\
\text { diente físicamente }\end{array}$ & $14(43,75 \%)$ \\
\hline 4 & Discapacidad severa. Aún capaz de caminar o bipedestar sin asistencia & $3(9,37 \%)$ \\
\hline 5 & En silla de ruedas o en cama si no es asistido & $0 \quad(0 \%)$ \\
\hline
\end{tabular}

Escala Hoehn \& Yahr: Mediante este instrumento se clasificó a la mayoría de pacientes como compromiso bilateral, pero indepedientes físicamente (Estadíos 2 y 3 ), tal como se muestra en la Tabla 2.

Trastorno conductual del sueño REM: Nueve de 32 pacientes $(28,12 \%)$ presentaban historia compatible con al menos un episodio significativo en el último mes. Cinco de ellos recibían tratamiento sintomático con clonazepam, indicado por el especialista en nivel secundario. Ninguno recibía melatonina.

Depresión: Al aplicar la escala GDS-15, nueve pacientes calificaban para depresión probable $(5 \mathrm{a}$ 9 puntos), y 5 presentaban depresión establecida ( $\geq 10$ puntos), tres de ellos mantenían tratamiento farmacológico por depresión previo a este estudio. Con más frecuencia respondieron afirmativamente a sensación de impotencia, aislamiento social, abandono de intereses y falta de energía. Ninguno de los pacientes recibía psicoterapia.

Deterioro cognitivo: Se aplicó el test de MoCA, agregando 2 puntos a aquellos con menos de 12 años de estudios, obteniendo una media de $21 \pm$ $4,95$ (10-28 puntos). Nueve ( $28,12 \%)$ lograron un puntaje $<20$, todos ellos con menos de 6 años de educación escolar, manteniendo $<20$ puntos al compensar con 3 puntos por escolaridad.

Escala de sintomas no motores (SNM): La media de esta cohorte fue 90,09 \pm DE: 43 (14174 puntos). De acuerdo al puntaje total de la escala, al determinar el nivel de carga de SNM, $20(62,5 \%)$ presentaban carga muy severa $(\geq 71$ puntos), 8 severa (25\%) (41-70 puntos), 3 carga moderada (9,3\%) (21-40 puntos), y 1 Leve $(3,1 \%)$ (1-20 puntos).

En la Tabla 3 se puede observar el número de pacientes que categorizaron cada síntoma abordado por la escala como "moderado" o "grave" y con presentación "frecuente" o "muy frecuente".

Descontrol de impulsos: De los 32 pacientes, uno presentaba descontrol de impulsos tipo ludopatía, un paciente presentaba craving, y en 1 caso se identificó punding.

Disregulación dopaminérgica: Se identificó 1 paciente con posible disregulación dopaminérgica.

\section{Discusión}

Este es, a nuestro entender, el primer estudio de evaluación de SNM y descontrol de impulsos en individuos con EP realizado por médicos de APS utilizando este tipo de escalas en Chile. Nos parece trascendental compartir estos resultados, buscando incitar a nuestros colegas a replicar y mejorar este tipo de estudios y estrategias.

El grupo de pacientes evaluados en el presente trabajo representa una población de adultos mayores, en muchos casos con educación escolar incompleta, y con escasa red de apoyo, factores que deben ser considerados al momento interpretar los datos y planificar estrategias de atención en la red de salud.

Entre las comorbilidades destacan, hipertensión arterial, patología cardiaca, artrosis, síndrome de hombro doloroso, hipoacusia y patología prostática. Esta observación refleja una oportunidad de intervención por parte del médico. Por ejemplo, los antihipertensivos y patología cardiaca pueden 
Tabla 3. Escala de síntomas no motores

\begin{tabular}{|c|c|c|c|}
\hline Dominio & Ítem & $\mathbf{n}^{*}$ & $(\%)$ \\
\hline \multirow[t]{2}{*}{ Cardiovascular/caídas } & 1. Ortostatismo & 3 & 9,38 \\
\hline & 2. Lipotimia/síncope & 0 & 0 \\
\hline \multirow[t]{4}{*}{ Sueño/fatiga } & 3. Somnolencia diurna & 11 & 34,38 \\
\hline & 4. Fatiga & 16 & 50 \\
\hline & 5. Insomnio & 10 & 31,25 \\
\hline & 6. Piernas inquietas & 12 & 37,5 \\
\hline \multirow[t]{6}{*}{ Ánimo/apatía } & 7. Pérdida de interés en lo que lo rodea & 6 & 18,75 \\
\hline & 8. Falta de motivación & 9 & 28,13 \\
\hline & 9. Preocupación/nerviosismo & 13 & 40,63 \\
\hline & 10. Parece estar triste & 9 & 28,13 \\
\hline & 11. Ánimo aplanado & 14 & 43,75 \\
\hline & 12. Dificultad en sentir placer & 7 & 21,88 \\
\hline \multirow[t]{3}{*}{ Problemas perceptivos } & 13. Alucinaciones & 3 & 9,38 \\
\hline & 14. Ilusiones & 3 & 9,38 \\
\hline & 15. Visión doble & 0 & 0 \\
\hline \multirow[t]{3}{*}{ Atención/memoria } & 16. Problemas de concentración & 7 & 21,875 \\
\hline & 17. Olvida hechos recientes & 11 & 34,38 \\
\hline & 18. Olvida hacer cosas & 3 & 9,38 \\
\hline \multirow[t]{3}{*}{ Tracto gastrointestinal } & 19. Salivación & 4 & 12,5 \\
\hline & 20. Dificultad en deglución & 7 & 21,88 \\
\hline & 21. Estreñimiento & 10 & 31,25 \\
\hline \multirow[t]{3}{*}{ Función urinaria } & 22. Urgencia miccional & 15 & 46,88 \\
\hline & 23. Frecuencia miccional & 8 & 25 \\
\hline & 24. Nicturia & 17 & 53,13 \\
\hline \multirow[t]{2}{*}{ Función sexual } & 25. Interés sexual alterado & 6 & 18,75 \\
\hline & 26. Dificultad en tener relaciones sexuales & 6 & 18,75 \\
\hline \multirow[t]{4}{*}{ Miscelánea } & 27. Dolor & 8 & 25 \\
\hline & 28. Alteración del gusto u olfato & 10 & 31,25 \\
\hline & 29. Cambio en el peso & 4 & 12,5 \\
\hline & 30. Sudoración excesiva & 2 & 6,25 \\
\hline
\end{tabular}

*Número de pacientes que reportaron sus síntomas como moderados o severos y con una frecuencia de frecuentes o muy frecuentes en cada ítem de los distintos dominios de la escala de síntomas no motores. 
exacerbar o gatillar hipotensión ortostática, siendo fundamental su monitorización a través de la toma de presión en decúbito y bipedestación. Se deben implementar estrategias, como ayudas técnicas, en pacientes con riesgo de caídas y uso de anticoagulantes. El manejo individualizado del dolor y rehabilitación en aquellos individuos con artrosis y síndrome de hombro doloroso puede tener un impacto en la movilidad, marcha, ánimo y sueño, teniendo precaución con el uso de opioides. La adecuada derivación de pacientes con hipoacusia a otorrinolaringología, puede tener un impacto beneficioso en el proceso de comunicación, el cual ya es afectado por la disartria e hipofonía, en algunos casos. La monitorización de patología prostática permite ayudar a distinguir de la sintomatología genitourinaria propia de la EP, además de pesquisar cuadros de retención urinaria que pueden ser gatillados por el uso de fármacos con efecto anticolinérgico como la amantadina.

Pesquisamos en este grupo de pacientes una sub-utilización del recurso de rehabilitación física, siendo esta última un pilar fundamental en el manejo no farmacológico, por ejemplo en problemas de marcha y equilibrio.

Destacamos la importante carga de SNM en esta población. Sobresaliendo los trastornos del sueño, piernas inquietas, trastorno del ánimo, síntomas gastrointestinales y genito-urinarios. Posibles intervenciones para estos problemas son las medidas de higiene de sueño, uso de melatonina en el trastorno conductual del sueño REM o pesquisa de causas secundarias de piernas inquietas (por ejemplo, hipotiroidismo o ferropenia). El manejo dietario e inicio de laxantes osmóticos permiten aliviar la constipación.

El screening de depresión, permitió identificar nuevos casos, sugiriendo un sub-diagnóstico en este grupo de pacientes. En tanto, el test de MoCA permitió identificar sujetos con compromiso cognitivo significativo, generando un espacio de intervención, en ambas problemáticas, por un equipo interdisciplinario.

El bajo número de pacientes afectados por alucinaciones, puede estar determinado por el menor uso de gatillantes como trihexifenidilo, amantadina, o agonistas dopaminérgicos. El rol en APS es descartar causas secundarias, por ejemplo infecciones intercurrentes. Al igual que la presencia de síntomas de descontrol de impulsos, estos deben ser informados oportunamente al médico especialista. Una explicación para la baja frecuencia de descontrol de impulsos en esta población es la edad avanzada al diagnóstico y el escaso uso de agonistas dopaminérgicos, o su uso en dosis bajas.

Creemos que una fortaleza de nuestro trabajo es el tipo de población. Evaluar SNM en pacientes atendidos en niveles terciarios o centros de atención especializada, posiblemente tiende a sobreestimar la frecuencia de presentación y su severidad. En nuestro trabajo al tratarse de una población no seleccionada del nivel primario de salud, disminuimos este sesgo.

Destacamos que nuestro protocolo es fácilmente replicable por médicos de atención primaria, y creemos que brinda herramientas simples pero importantes para mejorar la atención de pacientes con EP por parte del médico general o familiar.

Entre las debilidades de este trabajo destacamos que la población de pacientes con EP es pequeña en este centro de salud y por lo tanto, a pesar de ser representativa, nuestra muestra es reducida y no tiene potencia estadística para lograr resultados significativos al estratificar por subgrupos y realizar estudios de correlación.

Otra debilidad en nuestro estudio es la exclusión de pacientes pertenecientes al programa de dependencia severa. Es reconocido que pacientes en etapas más avanzada presentan mayor carga de síntomas motores y no-motores, por lo tanto creemos estar subestimando la severidad y frecuencia de estos síntomas en la población de pacientes con Párkinson de este centro de salud.

Si bien la escala de SNM no ha sido validada en población chilena en su versión validada en español, es una herramienta ampliamente utilizada por médicos especialistas y sub-especialistas con fin de pesquisa y monitoreo en nuestro País, y como mencionamos está validada para su uso en atención primaria. En el caso de la escala de depresión geriátrica a la fecha no cuenta con un estudio de validación nacional; sin embargo, es ampliamente utilizada en atención primaria y sugerida por el Ministerio de Salud de Chile para ser aplicada en el examen anual de medicina preventiva del adulto mayor a nivel primario.

Concluimos que, utilizando herramientas adecuadas y de fácil implementación, el médico de atención primaria juega un papel determinante en la monitorización y manejo de los aspectos no motores de la EP, facilitando además el trabajo 
colaborativo con médicos especialistas. Los resultados de este trabajo apoyan la necesidad de una atención integral transdisciplinaria e interconectada entre los diferentes niveles de atención.

Agradecimientos: Especial agradecimiento al Servicio de Neurología de Adultos del Hospital San Borja-Arriarán por su trabajo y rol en la capacitación de médicos de atención primaria.

\section{Referencias}

1. Tysnes OB, Storstein A. Epidemiology of Parkinson's disease. J Neural Transm 2017; 124 (8): 901-5. doi:10.1007/s00702-017-1686-y.

2. Pedro CC, Magdalena JC, Violeta DT, Juri C. Mortalidad por enfermedad de Parkinson en Chile. Rev Med Chile 2013; 141 (3): 327-31. doi: 10.4067/S003498872013000300007.

3. Hughes AJ, Daniel SE, Ben-Shlomo Y, Lees AJ. The accuracy of diagnosis of parkinsonian syndromes in a specialist movement disorder service. Brain 2002; 125 (4): 861-70. http://www.embase.com/search/results?subaction=viewrecord\&from=export\&id=L34279780.

4. Wu SL, Liscic RM, Kim S, Sorbi S, Yang YH. Nonmotor Symptoms of Parkinson's Disease. Parkinsons Dis 2017; 2017: 10-11. doi: 10.1155/2017/4382518.

5. Durcan R, Wiblin L, Lawson RA, Khoo TK, Yarnall AJ, Duncan GW, et al. Prevalence and duration of non-motor symptoms in prodromal Parkinson's disease. Eur J Neurol 2019; 26 (7): 979-85. doi: 10.1111/ene.13919.

6. Antonini A, Barone P, Marconi R, Morgante L, Zappulla S, Pontieri FE, et al. The progression of non-motor symptoms in Parkinson's disease and their contribution to motor disability and quality of life. J Neurol 2012; 259 (12): 2621-31. doi: 10.1007/s00415-012-6557-8.

7. Martínez-Martin P, Schapira AH, Stocchi F, Sethi K, Odin P, MacPhee G, et al. Prevalence of nonmotor symptoms in Parkinson's disease in an international setting; study using nonmotor symptoms questionnaire in 545 patients. Mov Disord 2007; 22 (11): 1623-9. doi: 10.1002/mds.21586.

8. Martínez-Martin P, Rodríguez-Blazquez C, Kurtis MM, Chaudhuri KR. The impact of non-motor symptoms on health-related quality of life of patients with Parkinson's disease. Mov Disord 2011; 26 (3): 399-406. doi: 10.1002/ mds.23462.

9. Thenganatt MA, Jankovic J. Parkinson disease subtypes. JAMA Neurol 2014; 71 (4): 499-504. doi: 10.1001/jamaneurol.2013.6233.
10. Sauerbier A, Jenner P, Todorova A, Chaudhuri KR. Non motor subtypes and Parkinson's disease. Park Relat Disord 2016; 22: S41-6. doi: 10.1016/j.parkreldis.2015.09.027.

11. Erro R, Vitale C, Amboni M, Picillo M, Moccia M, Longo K, et al. The Heterogeneity of Early Parkinson's Disease: A Cluster Analysis on Newly Diagnosed Untreated Patients. PLoS One 2013; 8 (8): 1-8. doi: 10.1371/journal.pone.0070244.

12. Weintraub D, David AS, Evans AH, Grant JE, Stacy M. Clinical spectrum of impulse control disorders in Parkinson's disease. Mov Disord 2015;30(2):121-127. doi: 10.1002/mds.26016.

13. Gatto EM, Aldinio V. Impulse Control Disorders in Parkinson's Disease. A Brief and Comprehensive Review. Front Neurol 2019; 10 (April). doi: 10.3389/ fneur.2019.00351.

14. Ávila A, Cardona X, Bello J, Maho P, Sastre F, Martín-Baranera M. Trastornos del control de los impulsos y punding en la enfermedad. Neurologia 2011; 26 (3): 166-72. doi: 10.1016/j.nrl.2010.09.007.

15. O’Sullivan SS, Evans AH, Lees AJ. Dopamine dysregulation syndrome: An overview of its epidemiology, mechanisms and management. CNS Drugs 2009; 23 (2): 157-70. doi: 10.2165/00023210-200923020-00005.

16. Hoehn MM, Yahr MD. Parkinsonism: onset, progression, and mortality. 1967; 17(May).

17. Goetz CG, Poewe W, Rascol O, Sampaio C, Stebbins GT, Counsell C, et al. Movement Disorder Society Task Force report on the Hoehn and Yahr staging scale: Status and recommendations. Mov Disord 2004; 19 (9): 1020-8. doi: 10.1002/mds.20213.

18. Gill DJ, Freshman A, Blender JA, Ravina B. The Montreal Cognitive Assessment as a screening tool for cognitive impairment in Parkinson's disease. Mov Disord 2008; 23 (7): 1043-6. doi: 10.1002/mds.22017.

19. Dalrymple-Alford JC, MacAskill MR, Nakas CT, Livingston L, Graham C, Crucian GP, et al. The MoCA: Well-suited screen for cognitive impairment in Parkinson disease. Neurology 2010; 75 (19): 1717-25. doi: 10.1212/WNL.0b013e3181fc29c9.

20. Zadikoff C, Fox SH, Tang-Wai DF, Thomsen T, de Bie RM, Wadia P, et al. A comparison of the mini mental state exam to the Montreal cognitive assessment in identifying cognitive deficits in Parkinson's disease. Mov Disord 2008; 23 (2): 297-9. doi: 10.1002/ mds. 21837.

21. Delgado C, Araneda A, Behrens MI. Validation of the Spanish-language version of the Montreal Cognitive Assessment test in adults older than 60 years. Neurologia. 2019; 34 (6): 376-385. doi: 10.1016/j.nrl.2017.01.013. 
22. Ortega Orcos R, Salinero Fort MA, Kazemzadeh Khajoui A, Vidal Aparicio S, De Dios Del Valle R. Validación de la versión española de 5 y 15 ítems de la Escala de Depresión Geriátrica en personas mayores en Atención Primaria. Rev Clin Esp 2007; 207 (11): 559-562. doi: 10.1016/S0014-2565(07)73477-X.

23. Weintraub D, Oehlberg KA, Katz IR, Stern MB. Test characteristics of the 15-item Geriatric Depression Scale and Hamilton Depression Rating Scale in Parkinson disease. Am J Geriatr Psychiatry 2006; 14 (2): 169-75. doi: 10.1097/01.JGP.0000192488.66049.4b.

24. Meara J, Mitchelmore E, Hobson P. Use of the GDS-15 geriatric depression scale as a screening instrument for depressive symptomatology in patients with Parkinson's disease and their carers in the community. Age Ageing. 1999;28(1):35-38. doi: 10.1093/ageing/28.1.35

25. Chaudhuri KR, Martínez-Martin P, Brown RG, Sethi K, Stocchi F, Odin P, et al. The metric properties of a novel non-motor symptoms scale for Parkinson's disease: Results from an international pilot study. Mov Disord 2007; 22 (13): 1901-11. doi: 10.1002/mds.21596.

26. Martínez-Martin P, Rodríguez-Blazquez C, Abe K,
Bhattacharyya KB, Bloem BR, Carod-Artal FJ, et al. International study on the psychometric attributes of the Non-Motor Symptoms Scale in Parkinson disease. Neurology 2009; 73 (19): 1584-91. doi: 10.1212/WNL. 0b013e3181c0d416.

27. Todorova A, Jenner P, Ray Chaudhuri K. Non-motor parkinson's: Integral to motor parkinson's, yet often neglected. Pract Neurol 2014; 14 (5): 310-22. doi: 10.1136/ practneurol-2013-000741.

28. Ray Chaudhuri K, Rojo JM, Schapira AH, Brooks DJ, Stocchi F, Odin P, et al. A Proposal for a Comprehensive Grading of Parkinson's Disease Severity Combining Motor and Non-Motor Assessments: Meeting an Unmet Need. PLoS One 2013; 8 (2): 1-7. doi: 10.1371/journal. pone.0057221.

29. Neikrug AB, Ancoli-Israel S. Diagnostic tools for REM sleep behavior disorder. Sleep Med Rev 2012; 16 (5): 415-29. doi: 10.1016/j.smrv.2011.08.004.

30. Litvinenko IV, Krasakov IV, Tihomirova OV. Sleep disorders in patients with dementia in Parkinson's disease. Zhurnal Nevrol i Psihiatr Im SS Korsakova 2011; 111 (9): 37-42. doi: 10.2165/00023210-200115040-00002. 